\title{
TESTES DE RACIONALIDADE PARA LOTERIAS NO BRASIL*
}

\author{
Marcos A. M. Lima ${ }^{\S}$ \\ Marcelo Resende ${ }^{\infty}$
}

\begin{abstract}
RESUMO
O artigo investiga a prevalência de expectativas racionais no caso de duas loterias brasileiras (Quina e MegaSena). A estratégia de teste se relaciona com uma condição de ortogonalidade entre o erro condicional de previsão e o conjunto de informação. Especificamente, o resíduo de uma equação para o preço líquido de um bilhete de loteria deveria ser não correlacionado com as vendas. Os resultados favoreceram a hipótese de expectativas racionais somente no caso da Mega-Sena que é sujeita a ampla cobertura da mídia. Claramente a Quina está associada a um perfil distinto de apostadores.
\end{abstract}

Palavras-chave: loteria, expectativas racionais.

\begin{abstract}
The paper investigates the prevalence of rational expectations in the case of two Brazilian lotteries (Quina and Mega-Sena). The testing strategy relates to an orthogonality condition between the conditional forecast error and the information set. Specifically, the residual of a equation for net price of a lottery ticket should be uncorrelated with sales The results favoured the rational expectations hypothesis only in the case of the Mega-Sena that is subject to broad media coverage. Clearly the Quina lottery is associated with a diferent profile of betters.
\end{abstract}

Key words: lotteries, rational expectations.

JEL classification: D12, D84.

* Os autores agradecem os comentários de dois pareceristas a uma versão anterior do trabalho, mas os eventuais erros remanescentes são de nossa responsabilidade.

$\S \quad$ Faculdade de Economia e Finanças - IBMEC-RJ. Av. Rio Branco 108, Centro, 20.040-001, Rio de Janeiro-RJ, Brazil. Email: mamdl@uol.com.br.

a Instituto de Economia, Universidade Federal do Rio de Janeiro. Av. Pasteur 250, Urca, 22290-240, Rio de Janeiro-RJ, Brazil. Email:mresende@ie.ufrj.br.

Recebido em outubro de 2004. Aceito em novembro de 2005. 


\section{INTRODUÇÃO}

A difusão de mecanismos institucionalizados de loterias tem sido recentemente expandida nos Estados Unidos e na Europa e, conseqüentemente, tem se ampliado o interesse na análise econômica correspondente (ver Walker, 1998 e Walker e Young, 2001, para resenhas).

No caso brasileiro, marcado por fortes desigualdades na distribuição de renda, não se pode deixar de notar o enorme fascínio causado pelos grandes acúmulos de prêmios e pode-se observar uma crescente proliferação de diferentes modalidades de loterias. Em particular, observa-se a redução da importância das loterias esportivas tradicionais que foram sobrepujadas por diferentes formas de loterias de números ou variantes com prêmios instantâneos ("raspadinhas"). Uma primeira constatação importante é que os montantes envolvidos são não desprezíveis e, portanto, uma análise econômica dessa atividade é oportuna, especialmente no caso brasileiro.

Em linhas gerais, as loterias podem ser caracterizadas em termos de aspectos relativos às dimensões de oferta e demanda. No primeiro caso, o foco dos estudos está no desenho ótimo do mecanismo de loteria do ponto de vista do provedor. Vale dizer, em que medida as modalidades de loteria atendem ao objetivo de maximização de receita. Com efeito, em uma economia como a brasileira, que já está sujeita a uma das maiores cargas tributárias do mundo, a loteria acaba por funcionar como um mecanismo de taxação voluntária que tem um papel relevante para viabilizar repasses para diferentes boas causas. Mais recentemente tem se observado no Brasil uma política ativa de expansão de diferentes loterias por parte das entidades estaduais e federais envolvidas, quer no conjunto de modalidades existentes, quer na freqüência dos sorteios. Dentre alguns estudos pelo lado da oferta merecem menção os trabalhos de Farrell e Walker (1998) e Walker (2001) para o Reino Unido e de Cook e Clofelter (1993) para os Estados Unidos.

Uma segunda vertente da literatura empírica, mais relacionada ao presente trabalho, investiga diferentes aspectos associados à demanda por loterias. Nesta linha destacam-se estudos que procuram detectar vício no comportamento de demanda por loterias, reforçados, em particular, por grandes acúmulos de prêmios ("rollover"), como indicado por Farrell et al. (1999), e ainda testes de racionalidade, que enfatizam a aderência (ainda que implícita) do comportamento dos jogadores ao valor esperado associado à compra de um bilhete de loteria (ver Scott e Gulley, 1995 e Forrest et al., 2000). Prima facie, poder-se-ia questionar que a noção de racionalidade poderia ser restritiva no contexto de loterias, na medida em que muitos jogadores poderiam obter um prazer relacionado ao simples ato de jogar que seja em parte dissociado da expectativa objetiva de ganho financeiro. Conlisk (1993), por exemplo, levanta tal linha de argumentação para motivar o jogo em cenários atuarialmente desfavoráveis. Tais observações, contudo, não invalidam necessariamente alguma noção de racionalidade. De fato, parece existir um número significativo de indivíduos que só jogam quando o prêmio está acumulado, e a ocorrência de prêmios acumulados não é rara como o seria o caso se os números fossem escolhidos de forma aleatória. Farrell et al. (2000) enfatizam o papel da escolha consciente de números no acúmulo freqüente de prêmios. Assim sendo, o aumento do valor esperado do bilhete nessas ocasiões pode estar positivamente associado ao aumento de vendas que freqüentemente é induzido quando os prêmios se acumulam significativamente.

A hipótese de expectativas racionais assume que os agentes (ainda que de forma intuitiva) não cometem erros de previsão sistemáticos. Neste sentido, uma condição chave refere-se à não correlação entre os erros de previsão condicionais e o conjunto de informação disponível. Tal condição de ortogonalidade é importante também do ponto de vista prático, já que a demanda por loterias tipicamente envolve agregação de apostadores que se defrontam com um mesmo valor esperado de bilhete e a hipótese de expectativas racionais seria central nessa aproximação (ver Walker, 1998). 
Vale ressaltar que a hipótese de expectativas racionais ampara-se na possibilidade dos agentes terem acesso (ainda que de forma intuitiva) ao modelo relevante para a decisão. No contexto de loterias, no qual se observa uma certa ausência de esforços de modelagem teórica mais profundos, segue-se que a análise empírica subseqüente se revestirá de um caráter exploratório.

O presente artigo pretende efetuar um esforço inicial de investigar economicamente as loterias de números no Brasil (Mega-Sena e Quina) no tocante a aspectos de racionalidade do comportamento da demanda. O trabalho está organizado da seguinte forma. Na segunda seção discute-se brevemente o procedimento a ser adotado para testar racionalidade. A terceira seção discute a base de dados, a construção das variáveis utilizadas no estudo e apresenta os resultados empíricos. A quarta seção traz os comentários finais e direções para pesquisas futuras.

\section{LOTERIAS DE NÚMEROS: ASPECTOS CONCEITUAIS}

O exame da racionalidade do comportamento de demanda por loterias está diretamente associado a relações bem definidas entre o valor esperado associado a um bilhete de loteria e o volume de vendas dos mesmos. Nesta seção, discutimos de forma sintética os elementos conceituais básicos envolvidos, mas contextualizados para o caso brasileiro. Especificamente, consideraremos a Mega-Sena (que prevê a escolha de 6 a 15 números dentre 60 números possíveis e cujo bilhete mais simples custa $R \$ 1,00$ ) e a Quina (que prevê a escolha de 5 a 8 números dentre 80 possíveis, e cujo bilhete mais simples custa $\mathrm{R} \$ 0,25)$.

\subsection{Valor esperado de um bilhete de loteria}

O primeiro autor a desenvolver uma expressão formal para o valor esperado de um bilhete de loteria foi Sprowls (1970). Esta expressão foi alterada por Lim (1995) e Scoggins (1995). No presente artigo será utilizada a expressão desenvolvida por estes dois últimos autores modificada, com a finalidade de se adequar às especificidades das loterias brasileiras (Quina e Mega-Sena, no caso). A expressão que será apresentada a seguir considera que os números são selecionados aleatoriamente pelos apostadores.

O valor do prêmio principal é igual à proporção ${ }^{2}$ (fixa) da arrecadação destinada a este prêmio, mais o prêmio acumulado do sorteio anterior (se houver). Além disso, no caso específico da Mega-Sena, uma proporção da arrecadação de cada sorteio é reservada com o intuito de ser acrescida ao prêmio principal dos sorteios de final zero.

Considerando $\mathrm{C}_{\mathrm{t}}$ como a arrecadação no período $\mathrm{t}, \mathrm{R}_{\mathrm{t}}$ como o montante acumulado do sorteio anterior, e $\pi_{6 \mathrm{t}}$ como a proporção da arrecadação destinada ao prêmio principal, define-se o valor do prêmio principal $\left(\mathrm{J}_{\mathrm{t}}\right)$ da seguinte forma:

1 Os referidos valores de bilhetes prevaleciam dentro do período amostral considerado.

2 No caso brasileiro, para a Mega-Sena o prêmio bruto corresponde a $46 \%$ da renda bruta (arrecadação). Deste montante:

$30 \%$ para os acertadores dos 6 números sorteados (Sena);

$25 \%$ para os acertadores de 5 dos 6 números sorteados (Quina);

$25 \%$ para os acertadores de 4 dos 6 números sorteados (Quadra);

$20 \%$ se destinam a integrar a premiação dos acertadores dos 6 números sorteados (Sena), nos concursos de final zero.

Para a Quina, a proporção da arrecadação destinada à premiação também é de 46\%, divididos como se segue:

$40 \%$ para os acertadores dos 5 números sorteados (Quina);

$30 \%$ para os acertadores de 4 dos 5 números sorteados (Quadra);

$30 \%$ para os acertadores de 3 dos 5 números sorteados (Terno). 


$$
J_{t}\left(\pi_{6 t}, R_{t} ; C_{t}\right)=R_{t}+\pi_{6 t} . C_{t}
$$

Nos sorteios de final zero para a Mega-Sena este valor será o seguinte:

$$
J_{t}\left(\pi_{6 t}, R_{t} ; C_{t}, R_{0}\right)=R_{t}+\pi_{6 t} \cdot C_{t}+R_{0}
$$

onde $\mathrm{R}_{0}$ representa o montante arrecadado que é guardado para os sorteios de final zero. Para a Quina, a expressão é a seguinte:

$$
J_{t}\left(\pi_{5 t}, R_{t} ; C_{t}\right)=R_{t}+\pi_{5 t} . C_{t}
$$

Uma vez definido o montante destinado ao prêmio principal, pode-se obter uma expressão para o valor esperado de um bilhete de loterias. Para tanto, é necessário utilizar as probabilidades de ganhar o prêmio máximo com um bilhete, $\mathrm{p}_{6} 1 /\left(\frac{60 !}{54 ! 6 !}\right)=1 / 50.063 .860(1 / 50.063 .860)$, para o caso da Mega-Sena, e $\mathrm{p}_{5}\left(1 /\left(\frac{80 !}{75 ! 5 !}\right)=1 / 24.040 .016\right)$, no caso da Quina. A probabilidade do prêmio acumular é igual à probabilidade de que nenhum dos jogadores vença o prêmio principal, que é $\left(1-p_{i}\right)^{C_{t}}, \mathrm{i}=5$ ou 6. Este cálculo leva em consideração o fato de que cada bilhete é vendido a $\mathrm{R} \$ 1,00$. No cálculo do valor esperado deve-se considerar também os prêmios menores. O valor esperado (V) pode ser representado pela seguinte expressão, apresentada em Farrell et al. (1999): ${ }^{3}$

$$
\begin{aligned}
& V\left(R_{t}, \pi_{6 t}, \pi_{j t}, p_{6} ; C_{t}\right)=\frac{\left\{\left[1-\left(1-p_{6}\right)^{\left.C_{t}\right]}\left[R_{t}+\pi_{6 t} \cdot C_{t}\right]+\sum_{j} \pi_{j t} C_{t}\right\}\right.}{C_{t}} \\
& V\left(R_{t}, \pi_{5 t}, \pi_{j t}, p_{5} ; C_{t}\right)=\frac{\left\{\left[1-\left(1-p_{5}\right)^{4 C}{ }_{t}\right]\left[R_{t}+\pi_{5 t} \cdot C_{t}\right]+\sum_{j} \pi_{j t} C_{t}\right\}}{C_{t}}
\end{aligned}
$$

A equação (4) representa o valor esperado de um bilhete de loterias da Mega-Sena, ${ }^{4}$ enquanto a equação (5) representa a mesma medida para a Quina. Os termos $\pi_{\mathrm{jt}}$ representam as parcelas da arrecadação destinadas aos prêmios menores (quina e quadra para a Mega-Sena, quadra e terno para a Quina). No anexo I encontram-se os gráficos dos valores esperados dos bilhetes das duas loterias para o período analisado neste artigo.

O preço líquido do bilhete de loteria (P) é dado por $\mathrm{R} \$ 1,00-\mathrm{V}$. O preço líquido do bilhete é a parcela do preço pago $(\mathrm{R} \$ 1,00)$ que não é retornada ao apostador na forma de prêmios. O valor esperado do bilhete a cada sorteio da Mega-Sena e Quina constitui um ingrediente-chave na implementação de testes de racionalidade para loterias que passam a ser discutidos na próxima subseção.

3 Durante o período considerado o bilhete básico da Mega-Sena custava $\mathrm{R} \$ 1$ ao passo que no caso da Quina o valor era de $\mathrm{R} \$ 0,25$ e assim os expoentes da probabilidade conjunta de acúmulo de prêmio seriam respectivamente $C_{t}$ e $4 C_{t}$ sob cenários de apostas básicas com 1 bilhete por apostador nas duas modalidades.

4 Para os sorteios de final zero a parte do valor esperado correspondente à expressão (1) é substituída pela equação (2). 


\subsection{Testes de expectativas racionais em loterias}

Nesta seção será apresentada a metodologia utilizada para testar a hipótese de expectativas racionais no mercado de loterias, ou seja, se os apostadores exibem expectativas racionais em suas decisões de apostar.

Para tanto, deve-se verificar se o apostador leva em consideração o valor esperado (e conseqüentemente o preço líquido) do bilhete em suas decisões sobre apostas. Para ter uma idéia do valor esperado do bilhete (equações (4) e (5)) o apostador deve ter informações sobre o prêmio acumulado, a probabilidade de ganhar o prêmio principal, a proporção da arrecadação destinada aos prêmios e a arrecadação. Com exceção da arrecadação, todas as demais variáveis são observáveis, ou seja, o apostador possui estas informações no momento em que decide sobre a aposta. Fica, portanto, faltando ao apostador obter informações sobre a arrecadação.

Se o apostador estimar corretamente a arrecadação, em média, ele estará estimando o valor esperado do bilhete corretamente. A questão a ser verificada passa então pela forma como os apostadores estimam o valor esperado (logo, o preço líquido) e a arrecadação. O teste para expectativas racionais consiste em verificar se os erros de previsão dos indivíduos possuem alguma relação com as vendas efetivas. Trata-se, portanto, de verificar uma condição de ortogonalidade relacionada à hipótese de expectativas racionais. ${ }^{5}$ Se os apostadores estimarem corretamente as vendas, os erros de previsão não deverão ser correlacionados com as vendas efetivas. Neste caso o mercado estará em equilíbrio de expectativas racionais, pois as expectativas dos apostadores coincidem, em média, com a arrecadação efetiva.

A implementação deste teste seguirá a metodologia desenvolvida por Scott e Gulley (1995) e utilizada também por Forrest et al. (2000). O método consiste de 2 estágios. No primeiro estágio é estimada uma equação para o preço líquido definida como se segue:

Preço Líquido $=\mathrm{R} \$ 1,00-\mathrm{V}=\mathrm{F}$ (constante, tendência, tendência ao quadrado, prêmio acumulado, prêmio acumulado ao quadrado, dummy para final de semana, dummy para sorteios de final zero, dummy para o sorteio com maior premiação)

A única ressalva a ser feita é de que as últimas duas dummies só foram utilizadas no caso da Mega-Sena. Os resíduos desta regressão são utilizados como regressores na equação em que a arrecadação é a variável dependente. Esta etapa consiste no segundo estágio. Ou seja:

\section{Arrecadação $=\mathrm{f}\left(\right.$ constante, resíduos do $1^{\circ}$ estágio $)$}

Os dois estágios são estimados por mínimos quadrados ordinários. No presente trabalho tomamos o cuidado adicional de considerar erros padrões robustos a heteroscedasticidade e a correlação serial associados à matriz de variância-covariância de Newey-West. É necessário ainda descartar a possibilidade de regressões espúrias, mediante a implementação de testes de raiz unitária para as diferentes variáveis consideradas e, eventualmente, de análise de co-integração caso não estacionariedades estejam presentes. O objetivo final do segundo estágio é analisar a significância estatística do coeficiente da variável VENDAS. Caso a hipótese de expectativas racionais seja válida, espera-se que o referido coeficiente seja não significativo.

5 Ver Engsted (2002) para uma resenha sobre análises empíricas da hipótese de expectativas racionais. 


\section{ANÁLISE EMPÍRICA}

\subsection{Descrição dos dados}

O trabalho utiliza dados anteriormente inexplorados referentes a loterias de números (MegaSena e Quina) fornecidos pela Gerência Nacional de Loterias-GELOT, da Caixa Econômica Federal. Dentre os dados disponíveis para cada sorteio consideramos, para o presente estudo, o total de arrecadação, o prêmio acumulado e as proporções do total arrecadado destinadas a cada faixa de prêmio (sena, quina e quadra no caso da Mega-Sena, e quina, quadra e terno no caso da Quina)). Os sorteios eram inicialmente semanais para a Mega-Sena (no período de 11/03/96 a 28/07/2001), mas posteriormente passaram a ocorrer 2 sorteios por semana (para os quais os dados disponibilizados referem-se ao período de 01/08/2001 a 10/11/2001). Assim, a amostra compreende 312 sorteios. Para a Quina, o período amostral abarca o período de 05/02/1998 a 10/11/2001, ocorrendo sorteios 2 vezes por semana até o dia 29/03/98. A partir de então há 3 sorteios semanais. Assim, o total de observações chega a 548 .

Os valores esperados dos bilhetes para a Quina e Mega-Sena são apresentados na Figura 1 a seguir.

Figura 1 - Valor esperado do bilhete de loteria

Valor Esperado (Mega-Sena)

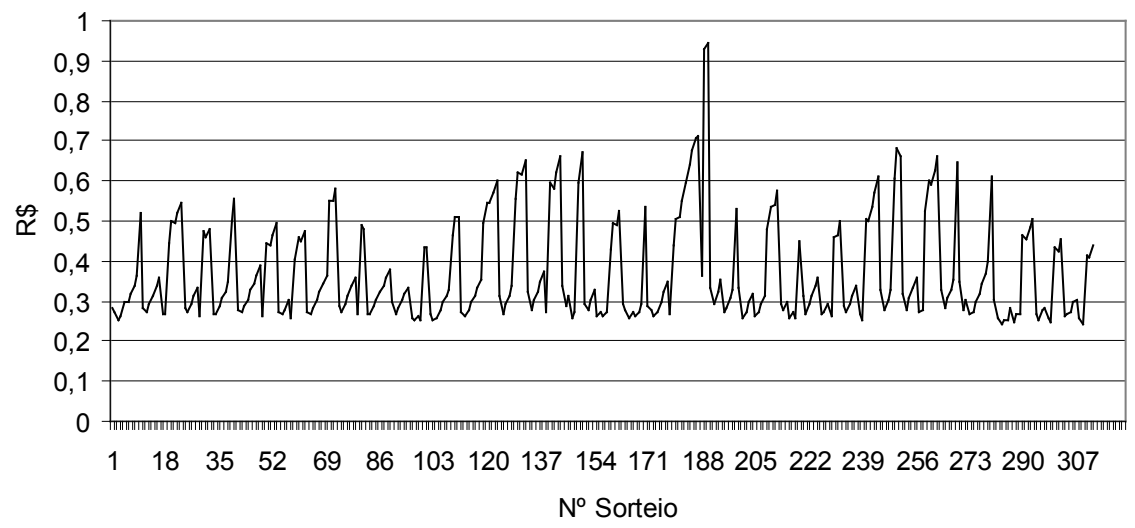

Valor Esperado (Quina)

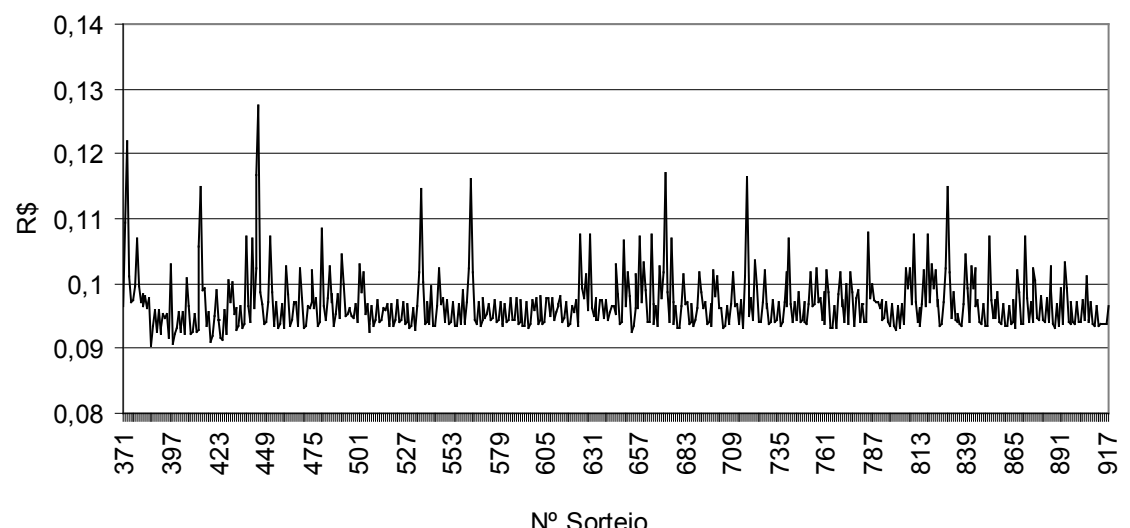


Vale mencionar que os valores esperados só mostram elevações importantes após sucessivos acúmulos de prêmios. Por fim, listamos a seguir as variáveis consideradas no procedimento de 2 estágios para Quina e Mega-Sena.

- p: preço líquido de um bilhete (com o número mínimo de dezenas) definido como o preço do bilhete menos o valor esperado do bilhete;

- ACUM: valor do prêmio acumulado;

- ACUM2: valor do prêmio acumulado elevado ao quadrado;

- TREND: variável de tendência temporal;

- TREND2: variável de tendência temporal em forma quadrática;

- VENDAS: valor das vendas para o concurso sob consideração;

- DQF: variável dummy que assume valor 1 nos sorteios realizados nas quartas-feiras e 0 caso contrário (usada somente na Mega-Sena);

- DFS: variável dummy que assume valor 1 nos sorteios realizados no final de semana e 0 caso contrário (usada somente na Quina);

- DZERO: variável dummy que assume valor 1 nos concursos com final zero e 0 caso contrário;

- DTRES: variável que assume valor 0 até o sorteio de 29/03/98 e valor 1 a partir de então.

A especificação aqui adotada é, portanto, semelhante àquela adotada por Forrest et al. (2000) com as devidas adaptações para o cálculo do valor esperado de um bilhete e da introdução de variáveis dummies condizentes com o desenho institucional brasileiro. Destaca-se, por exemplo, a variável DZERO, que capta o fato de sorteios com final zero terem uma alocação extra de prêmio e ainda as variáveis dummies refletindo a prevalência de múltiplos sorteios em cada semana.

Cabem, contudo, alguns comentários acerca da especificação adotada. A inclusão da tendência e da tendência ao quadrado é justificada por Forrest et al. (2000) pelo fato de as vendas de determinados bilhetes de loterias apresentarem uma tendência - crescente em um primeiro momento e depois declinante por diversas razões não explicadas pelas variáveis do modelo. Segundo esses autores, esta tendência observada pode influenciar o preço efetivo do bilhete. Por fim, merece menção a inclusão da variável de acúmulo de prêmio ao quadrado. Com efeito, tal especificação reflete a percepção, de resto intuitiva, de que o valor esperado do bilhete responderia de forma não linear relativamente àquele fenômeno já que então se observariam efeitos de vício relativamente mais intensos (ver, por exemplo, Farrell et al., 1999).

\subsection{Resultados empíricos}

Nesta seção reportamos os resultados do procedimento de 2 estágios, anteriormente mencionado, para a Quina e Mega-Sena. De início, procede-se a testes de raiz unitária para as variáveis potencialmente não estacionárias. Para a definição do modelo correto a ser utilizado nos testes de raiz unitária de Dickey-Fuller Aumentado (ADF) foi seguida a metodologia descrita por Enders (1995).

Primeiro definiu-se o número de defasagens. Para tanto, foi feito o teste ADF com um número grande de defasagens e observou-se se a última defasagem era estatisticamente significativa. Caso fosse, o modelo teria $\mathrm{N}$ defasagens, e caso não fosse, repetir-se-ia o teste com N-1 defasagens, e o mesmo procedimento seria realizado até encontrar o número correto de defasagens. Encontrado este número, partiu-se para a inclusão ou não de constante e tendência. ${ }^{6}$ Para tanto, estimou-se a

6 A tendência é avaliada como significativa ou não, segundo a tabela em Dickey e Fuller (1981), que pode ser também encontrada em Enders (1995, p. 223). 
equação com o número de defasagens definido anteriormente e com constante e tendência. Se a tendência fosse significativa, a equação conteria uma constante e uma tendência. Se a tendência não fosse significativa, retirar-se-ia a tendência e a equação seria estimada novamente para observar se a constante seria significativa. Se fosse, esta ficaria no modelo. Se não fosse, seria retirada. Em modelos lineares de séries de tempo, este tipo de metodologia parte do modelo geral para o específico.

Os resultados correspondentes aparecem no anexo e indicam que as variáveis são $\mathrm{I}(0)$ e assim não se precisa empreender testes de co-integração e nem se preocupar com a possibilidade de regressões espúrias. Assim sendo, pode-se fazer uso de procedimentos econométricos tradicionais. Os resultados da estimação econométrica para a Quina e Mega-Sena aparecem, respectivamente, nas Tabelas 1 e 2 apresentadas a seguir.

Tabela 1 - Quina $-1^{\circ}$ estágio

\begin{tabular}{lrccc}
\hline Variável & Coeficiente & Erro Padrão & Estatística-t & \multicolumn{1}{c}{ signif. } \\
\hline C & 0,153908 & 0,000233 & 660,3050 & 0,0000 \\
ACUM & $-2,83 \mathrm{E}-08$ & $6,22 \mathrm{E}-10$ & $-45,52997$ & 0,0000 \\
ACUM2 & $-6,31 \mathrm{E}-15$ & $1,07 \mathrm{E}-15$ & $-5,910268$ & 0,0000 \\
TREND & $-1,05 \mathrm{E}-05$ & $1,96 \mathrm{E}-06$ & $-5,356505$ & 0,0000 \\
TREND2 & $1,54 \mathrm{E}-08$ & $3,16 \mathrm{E}-09$ & 4,873556 & 0,0000 \\
DFS & $-0,002891$ & 0,000111 & $-26,07783$ & 0,0000 \\
DTRES & 0,003032 & 0,000314 & 9,652640 & 0,0000 \\
\hline$R^{2}$ & 0,919105 & estatística $\mathrm{F}=$ & 1024,451 \\
$\mathrm{R}^{2}$ Ajustado & 0,918208 & significância: & 0,000000 \\
\hline
\end{tabular}

Quina $-2^{\circ}$ estágio

\begin{tabular}{lcccc}
\hline Variável & Coeficiente & Erro Padrão & Estatística-t & signif. \\
\hline $\mathrm{C}$ & 0,004160 & 0,000226 & 18,38894 & 0,0000 \\
VENDAS & $-1,21 \mathrm{E}-09$ & $7,04 \mathrm{E}-11$ & $-17,16785$ & 0,0000 \\
\hline $\mathrm{R}^{2}$ & 0,384979 & \multicolumn{2}{c}{ estatística $\mathrm{F}=$} & 341,7744 \\
$\mathrm{R}^{2}$ Ajustado & 0,383852 & & significância:: & 0,0000 \\
\hline
\end{tabular}

Tabela 2 - Mega-Sena $-1^{\circ}$ estágio

\begin{tabular}{lcccc}
\hline Variável & Coeficiente & Erro Padrão & Estatística-t & signif. \\
\hline C & 0,744854 & 0,002678 & 278,1359 & 0,0000 \\
DZERO & $-0,040610$ & 0,003758 & $-10,80672$ & 0,0000 \\
DQF & 0,013928 & 0,003549 & 3,924163 & 0,0001 \\
ACUM & $-1,92 \mathrm{E}-08$ & $6,81 \mathrm{E}-10$ & $-28,10740$ & 0,0000 \\
ACUM2 & $1,49 \mathrm{E}-16$ & $3,62 \mathrm{E}-17$ & 4,125293 & 0,0000 \\
TREND & $-0,000103$ & $4,42 \mathrm{E}-05$ & $-2,329918$ & 0,0205 \\
TREND2 & $2,50 \mathrm{E}-07$ & $1,45 \mathrm{E}-07$ & 1,719886 & 0,0865 \\
\hline R $^{2}$ & 0,977588 & \multicolumn{3}{c}{ estatística $\mathrm{F}=$} \\
R $^{2}$ Ajustado & 0,977147 & \multicolumn{3}{c}{ significância: } \\
\hline
\end{tabular}

7 A escolha do número de defasagens nos testes de raiz unitária seguiu procedimento listado em Enders (1995), segundo o qual deve-se partir de um número grande de defasagens e estimar o modelo com um número cada vez menor de defasagens, até que o coeficiente da última defasagem seja estatisticamente significativo.

8 As estimativas foram implementadas com o software Eviews 5.0. 


\section{Mega-Sena $-2^{\circ}$ estágio}

\begin{tabular}{lccll}
\hline Variável & Coeficiente & Erro Padrão & Estatística-t & Signif. \\
\hline C & 0,006819 & 0,003876 & 1,759274 & 0,0795 \\
VENDAS & $-3,84 E-10$ & $2,55 E-10$ & $-1,504111$ & 0,1336 \\
\hline$R^{2}$ & 0,104783 & estatística & $F=$ & 36,28479 \\
R $^{2}$ Ajustado & 0,101895 & Significância: & 0,0000 \\
\hline
\end{tabular}

Os resultados obtidos diferem de acordo com o tipo de loteria. Para a Quina a condição de ortogonalidade é violada, já que o coeficiente da variável VENDAS no segundo estágio é fortemente significativo. Para a Mega-Sena, em contraste, observa-se resultado contrário e, portanto, consistente com a hipótese de expectativas racionais. Com efeito, esse resultado se alinha com a evidência anteriormente obtida para o Reino Unido em loteria semelhante. O resultado é sugestivo, já que ao contrário da Mega-Sena as outras modalidades de jogos não têm grande cobertura de mídia e, portanto, atraem um perfil distinto de apostadores.

\section{COMENTÁRIOS FINAIS}

Este trabalho procurou empreender um esforço inicial de análise econômica da atividade lotérica no caso brasileiro. Em particular, procuramos investigar a hipótese de comportamento racional. A evidência favoreceu a prevalência de expectativas racionais para a Mega-Sena, mas não para a Quina. No primeiro caso estamos obtendo resultado análogo àquele obtido para loteria semelhante no Reino Unido, indicando que mesmo que a atração por essa loteria seja em grande medida induzida por acúmulos de prêmios ter-se-ia implicitamente um comportamento racional.

Possíveis linhas de investigação futuras envolvem a detecção de padrões de vício na demanda por loterias que dependem do acúmulo de prêmios. Essa linha já foi iniciada por Farrell et al. (1999) para o Reino Unido. No caso brasileiro, a disponibilidade de dados por unidade da federação permite a investigação de questões não anteriormente consideradas na literatura.

Por fim, investigações de caráter mais estrutural não aparecem na literatura. Uma fundamentação teórica mais rigorosa da demanda por loterias seria desejável, contudo tais esforços extrapolam o escopo do presente artigo, que pode ser visto como uma investigação exploratória inicial em uma área ainda pouco explorada.

\section{REFERÊNCIAS}

Conlisk, J. The utility of gambling. Journal of Risk and Uncertainty, 6, p. 255-275, 1993.

Cook, P. J.; Clofelter, C. T. The peculiar scale economies of lotto. American Economic Review, 83, p. 634643, 1993.

Enders, W. Applied econometric time series. New York: John Wiley \& Sons Inc., 1995.

Engsted, T. Measures of fit for rational expectations models. Journal of Economic Surveys, 16, p. 569-589, 2002.

Farrell, L.; Hartley, R.; Lanot, G.; Walker, I. The demand for lotto: the role of conscious selection. Journal of Business \& Economics Statistics, 18, p. 228-241, 2000. 
Farrell, L.; Morgenworth, E.; Walker, I. A time series analysis of U.K. lottery sales: long and short run price elasticities. Oxford Bulletin of Economics and Statistics, 61, p. 513-526, 1999.

Farrell, L.; Walker, I. The welfare effects of lotto: evidence for the U.K. Journal of Public Economics, 72 , p. 99-120, 1998.

Forrest, D.; Gulley, O. D.; Simmons, R. Testing for rational expectations in the UK national lottery. Applied Economics, 32, p. 315-326, 2000.

Gulley, O. D.; Scott, F. A. The demand for wagering in state operated lotto games. National Tax Journal, 46, p. 13-22, 1993.

Lim, F. W. On the distribution of lotto. Australian National University, Working Paper, n. 282, 1995.

Scoggins, J. Lotto and expected net revenue. National Tax Journal, 48, p. 61-70, 1995.

Scott, F. A.; Gulley, O. D. Rationality and efficiency in lotto markets. Economic Inquiry, 33, p. 175-188, 1995.

Walker, I. The economic analysis of lotteries. Economic Policy, p. 359-401, 1998.

Walker, I.; Young, J. An economist guide to lottery design. Economic Journal, 111, p. 700-722, 2001. 


\section{ANEXo - TESTES DE RAIZ UNITÁRIA}

\section{Quina}

\begin{tabular}{lccccc}
\hline Variável & Termos da Equação & Número de & Estatística de teste & \multicolumn{2}{c}{ Valor Crítico } \\
\cline { 5 - 6 } & & Defasagens & (ADF) & $5 \%$ & $1 \%$ \\
\hline P & Constante & 5 & $-8,5252$ & $-2,8672$ & $-3,4448$ \\
ACUM & Constante & 0 & $-21,1568$ & $-2,8672$ & $-3,4448$ \\
ACUM2 & Constante & 0 & $-19,3594$ & $-2,8672$ & $-3,4448$ \\
VENDAS & Constante & 5 & $-6,9763$ & $-2,8672$ & $-3,4448$ \\
\hline
\end{tabular}

\section{Mega-Sena}

\begin{tabular}{lccccc}
\hline Variável & Termos da Equação & Número de & Estatística de teste & \multicolumn{2}{c}{ Valor Crítico } \\
\cline { 5 - 6 } & & Defasagens & $($ ADF) & $5 \%$ & $1 \%$ \\
\hline$P$ & Constante & 8 & $-4,3321$ & $-2,8712$ & $-3,4537$ \\
ACUM & Constante & 3 & $-7,8736$ & $-2,8711$ & $-3,4534$ \\
ACUM2 & Constante & 3 & $-6,2023$ & $-2,8711$ & $-3,4534$ \\
VENDAS & Constante & 1 & $-6,8792$ & $-2,8710$ & $-3,4533$ \\
\hline
\end{tabular}

